\title{
Ocula $a^{14}$
}

\section{Le città visibili. Per Giovanna Franci}

A cura di Ruggero Ragonese

Dicembre 2013 | www.ocula.it - DOI: 10.12977/ocula23

\section{Città o evento? \\ Rappresentare e costruire la città attraverso i Festival e i grandi eventi ${ }^{1}$}

di Elena Lorenzetto

Università di Bologna

lorenzetto.elena@gmail.com

\begin{abstract}
Le città si fanno sempre più evento: manifestazioni culturali, grandi eventi fieristici, appuntamenti sportivi internazionali, congressi politici. Un caso emblematico è costituito dal proliferare di festival culturali in città e territori d'ogni taglia. Che rapporto c'è tra questi eventi e le città? Lungi dall'essere semplice sede e allestimento di manifestazioni culturali, le città diventano soggetti attivi dell'evento organizzato fino a coincidere con esso. Che effetto producono queste forme di ibridazione tra città ed evento culturale? Che senso assumono per la città? In che modo contribuiscono al "fare città"? Attraverso un caso di studio specifico, si rifletterà su come l'organizzazione di eventi costituisce una nuova forma di rappresentazione della città e del territorio, nonché una nuova forma di città, che condensa in sé dimensione materiale $\mathrm{e}$ immateriale, morfologia e discorso, sistema e processo.
\end{abstract}

\section{Parole chiave}

Semiotica urbana, Grandi eventi organizzati, Rappresentazione, Mappa, Città.

\section{Sommario}

1. Introduzione

2. Il Festival delle Città Impresa

3. Il territorio del Festival delle Città Impresa

4. Il festival come strategia territoriale

5. Il caso di "Comodamente. Città e Parole in Festival".

6. Conclusioni

Bibliografia

${ }^{1}$ Comunicazione presentata al Convegno "Le città visibili”, Bologna, Scuola Superiore di Studi Umanistici, 18-19 maggio 2010. 


\section{Ocula ${ }^{14}$}

Le città visibili. Per Giovanna Franci | Elena Lorenzetto, Città o evento?

\section{Introduzione}

Sempre più le città europee, e non solo, sono animate da una variegata realtà di grandi eventi organizzati, classificabili in diversi format: festival, kermesse, rassegne, convention, grandi eventi fieristici, saloni, grandi manifestazioni culturali o appuntamenti sportivi internazionali. Si tratta di eventi straordinari, di solito a cadenza annuale, di breve durata, e che coinvolgono l'intera città, e non sono circoscritti a un singolo spazio come un teatro o un auditorium. In questo modo, hanno delle forti ricadute sulla città, sia sul suo immaginario, sia sulla sua morfologia, spesso con un adattamento dell'architettura e dell'urbanistica all'evento. Questo non riguarda solo casi eclatanti e storici come Olimpiadi ed Expo, che stanno ridisegnando città come Londra e Milano, ma ha a che fare anche con quel proliferare di festival che ha conquistato negli ultimi anni l'Italia . Basti considerare che a Bologna è stato istituito il "Festival of Festivals" ed è anche nato un magazine dei festival italiani: "Fanatic About Festivals".

Numerosi sono gli studi che monitorano il loro impatto economico sul territorio, mentre, come notano Floris Langen e Beatrix Garcia (2009) nello loro analisi sulla letteratura sui grandi eventi, meno si è indagato sugli effetti indiretti e intangibili:

Some of economic impact studies identified the need for additional research on social impacts. For instance, a report on Manchester's Pillar Events by Jura Consultants (2006) notes a major research gap in understanding the indirect impacts of major events upon host communities, and point out that the intangible or less easily measured outcomes or outputs are 'often ignored or poorly dealt with' (Langen e Garcia 2009: 4).

Dunque, quali effetti producono queste forme di ibridazione tra città ed evento culturale? In che modo contribuiscono al "fare città"? Per esempio, quanto e come la Torino contemporanea è la Torino delle Olimpiadi invernali? Quanto Mantova è il Festival Letteratura nell'immaginario collettivo?

Come afferma Giulia Agusto "l'evento culturale festival, nella sua forma più complessa e multiforme, assume rilevanti significati nello sviluppo territoriale 'culture-driven" (Agusto 2008: 2) e funge da "strumento per imprimere effetti di rigenerazione territoriale e di rinnovamento urbano" (Ibidem). Quindi:

Per questo motivo, per evitare banalizzazioni, pur essendo consapevoli dei reali effetti prodotti dai festival è utile affiancare valutazioni di tipo economico a valutazioni di tipo non economico che prevedano indicatori per valutare variabili quale l'accrescimento della diversità culturale, della promozione urbana, dell'inclusione sociale e della qualità della vita dei residenti" (Agusto 2008: 7).

Attraverso l'analisi di un caso di studio, intendo mostrare come un evento di questo tipo agisca sulla città, sia sulla sua immagine che sulla sua morfo- 


\section{Ocula $\mathbf{a}^{14}$}

Le città visibili. Per Giovanna Franci | Elena Lorenzetto, Città o evento?

logia, tanto da costituire un'interessante e innovativa strategia sia di rappresentazione sia di progettazione urbana.

\section{Il Festival delle Città Impresa}

Il caso di studio che presento è stato scelto perché esemplare per indagare la strategia di city making: il Festival delle Città Impresa.

Il Festival delle Città Impresa è un "festival di approfondimento culturale", formula che l'economista della cultura Guido Guerzoni definisce "format di produzione culturale di breve e brevissima durata" (Guerzoni 2008: 79). Possiamo riassumerne come tratti salienti: la centralità della parola e del discorso come mezzi per veicolare messaggi, idee e riflessioni; la presenza di personalità note tali da attrarre pubblico (cfr. Agusto 2008); un pubblico che "cerca l'approfondimento assieme al valore esperienziale e alla trasmissione del sapere" (Guerzoni 2008: 79).

Il Festival delle Città Impresa, nato nel 2008, si svolge ogni anno ad aprile e propone cinque giorni di incontri, dibattiti, eventi che si svolgono in contemporanea in una rete di centri urbani del Nord Est d'Italia: come riportato nella campagna di comunicazione si tratta di "un'area che interessa sei province e tre regioni, Veneto, Friuli Venezia Giulia e Trentino Alto Adige”. È organizzato e promosso dalla rivista Nordesteuropa.it e dal Corriere della Sera. Il programma di eventi verte sulle trasformazioni sociali, culturali ed economiche avvenute e in corso nel Nord Est, discusse seguendo un tema, scelto per ogni edizione.

Va sottolineato innanzitutto che il territorio che ospita il Festival della Città Impresa, non corrisponde al prototipo di sede di manifestazioni culturali, che è quello di un comune di dimensioni medie (tra 40.000 e 180.000 abitanti), possibilmente con un centro storico ben concentrato. Poi un'altra peculiarità di questo caso è che i comuni che ospitano gli eventi sono centri di piccole dimensioni localizzati in diverse province del Triveneto, minoritari rispetto a grandi e medie città quali Verona, Padova, Trento .

Ciò che connota le sedi è l'identità di città-impresa: questo termine, ideato per il festival, designa quei centri urbani che simboleggiano, nella loro storia, cultura e fisionomia il Nordest. Come riporta il materiale promozionale del Festival: "sono protagonisti i centri urbani, simbolo della trasformazione del tessuto economico e sociale del Nordest", trasformazioni che hanno mutato l'area da agricola a terra diffusa di piccole e medie imprese.

Questo festival è un caso particolarmente significativo perché ha luogo in un territorio identificato dagli studi urbanistici come città diffusa. Questo termine, coniato dall'urbanista Francesco Indovina (cfr. Indovina et al. 1990) inizialmente proprio per descrivere l'area del Veneto centrale, indica un territorio esteso, fuori dalla città tradizionale, in cui sono dispersi, senza ordine né coesione, insediamenti, servizi, infrastrutture. Un fenomeno, che oltre al Triveneto interessa molte aree italiane ed europee, esito del cosiddetto sprawl. Ma ciò che definisce, secondo gli urbanisti, questi territori come città diffusa, oltre alla dispersione di insediamenti, è di essere usati e vissuti come fossero un'unica grande città estesa, sulla base di una quotidiana mobilità 


\section{Ocula $\mathbf{a}^{14}$}

Le città visibili. Per Giovanna Franci | Elena Lorenzetto, Città o evento?

automobilistica che non si rivolge a un solo centro, ma è reticolare. Non sono quindi più sempre pertinenti i confini amministrativi e geografici; piani urbanistici e mappe non bastano per restituirne la configurazione, che ha invece a che fare con gli usi, le pratiche e i flussi di persone, merci, denaro, informazioni. Il fenomeno della città dispersa rende evidente come il criterio della visibilità spaziale non sia più adeguato per restituire l'identità del territorio, una questione che Franco Farinelli (2003) riconosce come emergente del mondo contemporaneo e globalizzato. Fra l'altro, questi territori sono carenti di rappresentazioni e immagini di sé e sono stati molto spesso messi in scena come territori marginali, secondari rispetto alle città tradizionali.

A partire da questa prospettiva, intendo analizzare come il grande evento culturale, con la sua congerie di discorsi e testi, possa essere uno strumento per configurare un tale territorio. Lo svolgersi dell'evento è solo una delle sue componenti. Il festival coinvolge diversi livelli di pertinenza e piani di articolazione del senso, che vanno esplicitati:

- il livello delle pratiche, che include la programmazione e lo svolgimento degli eventi, ma anche l'organizzazione di queste stesse attività. Quest'ultima coinvolge una rete di attori, organizzatori, promotori, sponsor, che, come vedremo, concorre a configurare il territorio del festival;

- il livello della messa in discorso dell'evento e dei suoi contenuti, con un kit di materiale promozionale e informativo, costituito da testi, immagini, sito web, materiale audiovisivo (per esempio, comunicati stampa, manifesti pubblicitari, video promozionali, mappe e programma di eventi);

- la messa in scena di oggetti e spazi del territorio, con affissioni, allestimenti scenici, indicazioni stradali;

- coinvolge inoltre la traduzione di questi discorsi e pratiche in altri discorsi mediatici e non. Questi ultimi riguardano la produzione di articoli e servizi giornalistici, saggi e tutta una rete di discorsi di cui è il festival stesso l'oggetto, con un inscatolamento enunciativo tra discorso sul festival e discorso del festival.

È questo l'aspetto di interesse semiotico: che forme dell'espressione differenti concorrono a configurare la medesima immagine di territorio.

\section{Il territorio del Festival delle Città Impresa}

Vediamo quali sono i tratti figurativi e i temi su cui si basa questa forma di rappresentazione. La struttura con cui il festival articola il territorio è la rete: isotopia - oggi alquanto abusata e banalizzata - che coinvolge diverse dimensioni del festival. Il territorio si struttura in un palinsesto di attività unitario ma il cui svolgimento è diramato su diversi centri. Il materiale promozionale descrive il festival come "una manifestazione a rete", costituita da "una rete di eventi", ospitato da una "rete di centri urbani”. L'isotopia della rete è anche tema degli stessi dibattiti e conferenze, che parlano per esempio di "Rete di innovatori", oppure di "Sviluppo della rete infrastrutturale del Nord Est". Inoltre, una rete di enti locali pubblici e privati promuove e sponsorizza il festival. Di conseguenza, la rete viene al tempo stesso discussa, messa in pratica e rappresentata. 


\section{Ocula ${ }^{14}$}

Le città visibili. Per Giovanna Franci | Elena Lorenzetto, Città o evento?

In questa struttura i centri urbani si riconfigurano come i nodi. In ciascun centro la kermesse affronta un tema specifico che rispecchia l'identità della città ospitante ed è, al tempo stesso, la declinazione locale di un macrotema che unifica i luoghi in un'area sia tematica sia territoriale . Il logo del Festival (v. fig. 1) manifesta questa modellizzazione del territorio. Una foglia, elemento del territorio, è innervata in nodi che corrispondono alle sedi del Festival, in ciascuno dei quali è indicato il tema discusso negli eventi. Costituisce così una mappa del territorio-festival.

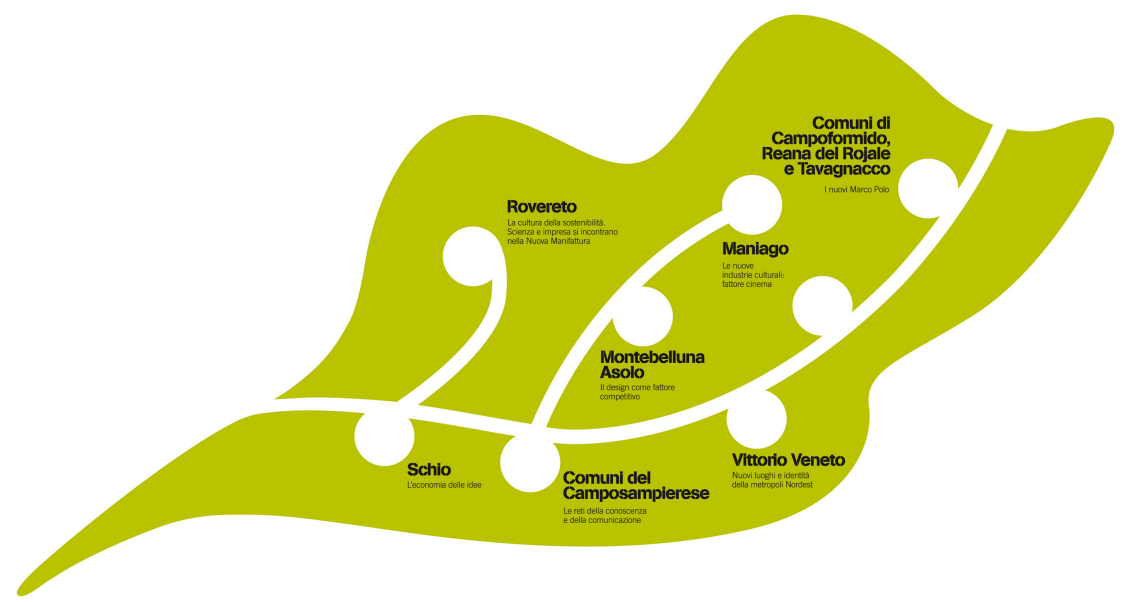

Figura 1. L’immagine dell'edizione 2010 del Festival delle Città Impresa.

Osserviamo anche la mappa del folder del programma che traccia i percorsi per raggiungere le sedi (v. fig. 2): se i confini regionali sono debolmente disegnati con un bordo grigio tenue, quelli provinciali non sono nemmeno rappresentati. I punti marcati sono i centri del festival e i nodi della mobilità che permettono l'interconnessione e che rendono possibile una configurazione a rete . Il territorio è rappresentato anche connesso con l'esterno, marcando la presenza di aeroporti. La piccola mappa in alto a sinistra inoltre lo posiziona entro il contesto italiano, costruendo così un punto di vista esterno rispetto al Nord Est e un destinatario outsider, inserito in una dimensione nazionale.

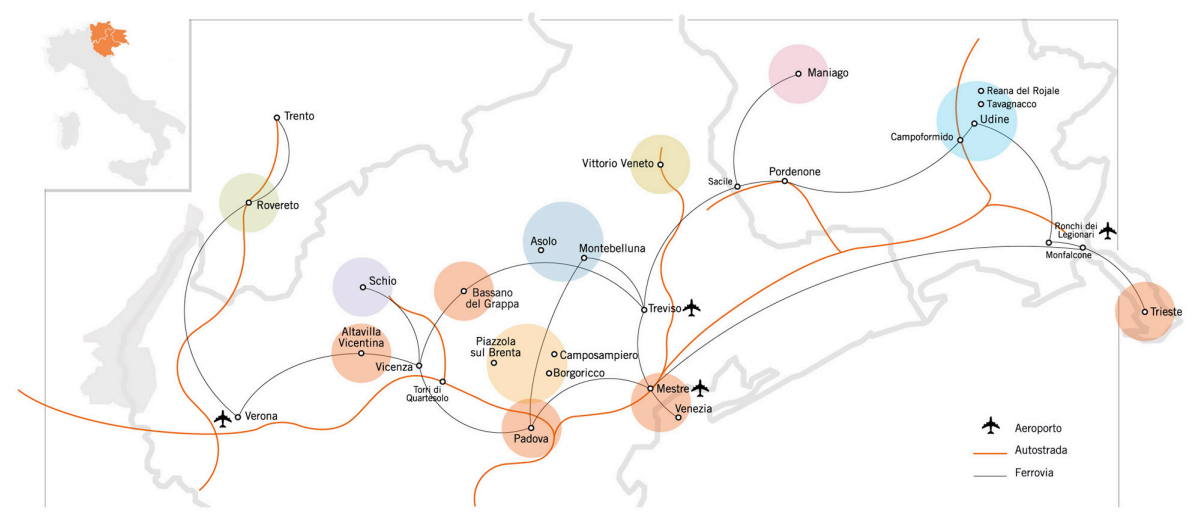

Figura 2. Mappa delle sedi del Festival 2010, inserita nel folder del programma. 


\section{Ocula $\mathbf{a}^{14}$}

Le città visibili. Per Giovanna Franci | Elena Lorenzetto, Città o evento?

Il territorio è dunque unificato sotto una medesima identità: il Nord Est. Quello che è interessante notare è che Nord Est non si definisce sulla base di confini comunali, provinciali o regionali, né attraverso piani urbanistici. Nord Est è innanzitutto un fenomeno economico divenuto paradigma, che il Festival promuove come "un modello europeo e mondiale di fare impresa", una forma che ha organizzato il territorio trasformandolo in una diffusione di piccole e medie imprese. In questo caso un modello economico diventa forma dell'espressione (della geografia) del territorio. Questa strategia è rilevabile nella nominazione stessa del festival: città-impresa, che esprime in modo tanto semplice quanto efficace l'operazione del festival, quella di usare il modello economico come criterio di pertinenza per definire e rappresentare il territorio.

Nord Est corrisponde così a quello che Greimas nel saggio "Per una semiotica topologica" (1976) chiama referente immaginario globale. Immaginario che è costruito in modo coerente attraverso l'integrazione di diversi oggetti semiotici: il logo, il programma del Festival, lo svolgersi degli eventi, i testi del festival. Valga come esempio il recital che l'attore Marco Paolini ha appositamente scritto e messo in scena per l'edizione 2010, dal titolo "Bisogna: la pellagra via sms". Lo spettacolo tratta in modo critico la trasformazione del territorio da terra della pellagra a città diffusa di capannoni, villette e rotonde ed è stato portato in scena con una mini-tournée nei luoghi del Festival. Inserito nella strategia dell'evento, anche questo contribuisce così a costruire l'immaginario del Nord Est.

Un altro tratto saliente di questa immagine è l'identità metropolitana, promossa addirittura da un libro intitolato "Manifesto per una Metropoli del Nordest", da cui è stato tratto uno spettacolo teatrale messo in scena nell'edizione 2009 del Festival. Come dichiarato nel materiale promozionale, il territorio "è visto come una grande area metropolitana di sette milioni di abitanti". Quindi, come attesta l'uso del verbo "vedere", Nord Est metropoli viene costruita sulla condivisione di una medesima percezione, più che sulla base di una rete di infrastrutture fisiche che connette la moltitudine di periferie, zone industriali, edge-cities che la punteggia. Questa strategia di costruzione di un modello "metropoli" è ben esemplificata in questa mappa (v. fig. 3). L'immagine fa parte del materiale promozionale dell'edizione 2010, che ha riflettuto sulle potenzialità che la cultura può avere per l'economia. Il titolo di questa mappa è "Mappa della metropoli culturale del Nord Est". Come si può vedere, i centri urbani del Nord Est sono connessi e interconnessi secondo i diversi settori dell'economia della cultura e dell'arte, quali il design, l'architettura, la moda, etc. Si può riconoscervi la celebre struttura della mappa del sistema di trasporto metropolitano di Londra (v. fig. 4), divenuto un simbolo della metropoli. Con una forte strategia di manipolazione questa forma viene importata e tradotta entro un sistema di riferimento differente, con un'altra scala ma soprattutto sulla base di un diverso criterio di pertinenza, quello economico: ad essere rappresentate non sono le connessioni fisiche, bensì le connessioni economiche. Importando la forma di metropolitana in un sistema differente, i diversi settori dell'economia vengono 


\section{Ocula ${ }^{14}$}

Le città visibili. Per Giovanna Franci | Elena Lorenzetto, Città o evento?

così risemantizzati in infrastrutture, capaci di mettere in relazione elementi $\mathrm{e}$ flussi del territorio, dando così struttura e coerenza di senso al territorio. Inoltre, una realtà urbanistica completamente differente rispetto al referente originario, la città di Londra, è interpretata come metropoli. Come effetto, si trasforma anche il concetto stesso di "metropolitano": non più un tratto formale dell'organizzazione del territorio, ma piuttosto uno stile, basato sulla mobilità e su un sistema di riferimento economico (cfr. Lorenzetto 2012).

Si può ravvisare quanto la funzione manipolante di questa operazione sia forte anche per un altro motivo. Oltre a definire il territorio come metropoli, ridefinisce anche lo stesso concetto di metropoli, promuovendo come "metropolitano" ciò che non lo è nel senso comune e nell'immaginario collettivo. Stiamo infatti parlando di un'area vasta, con forti carenze nella mobilità pubblica, nella governance del territorio e nella tutela del paesaggio, come questi scatti fotografici possono suggerire (v. figg. 5-6).

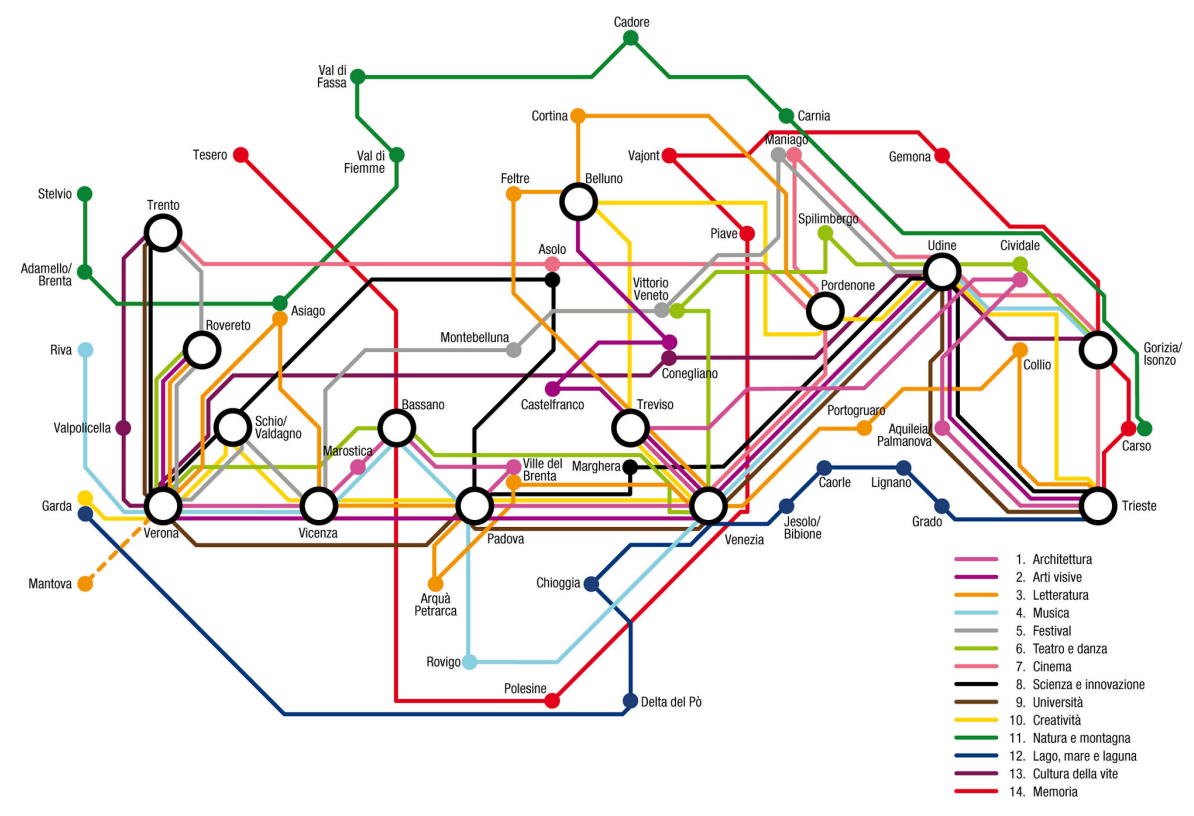

Figura 3. Mappa della Metropoli Culturali del Nord Est. 


\section{Ocula $a^{14}$}

Le città visibili. Per Giovanna Franci | Elena Lorenzetto, Città o evento?

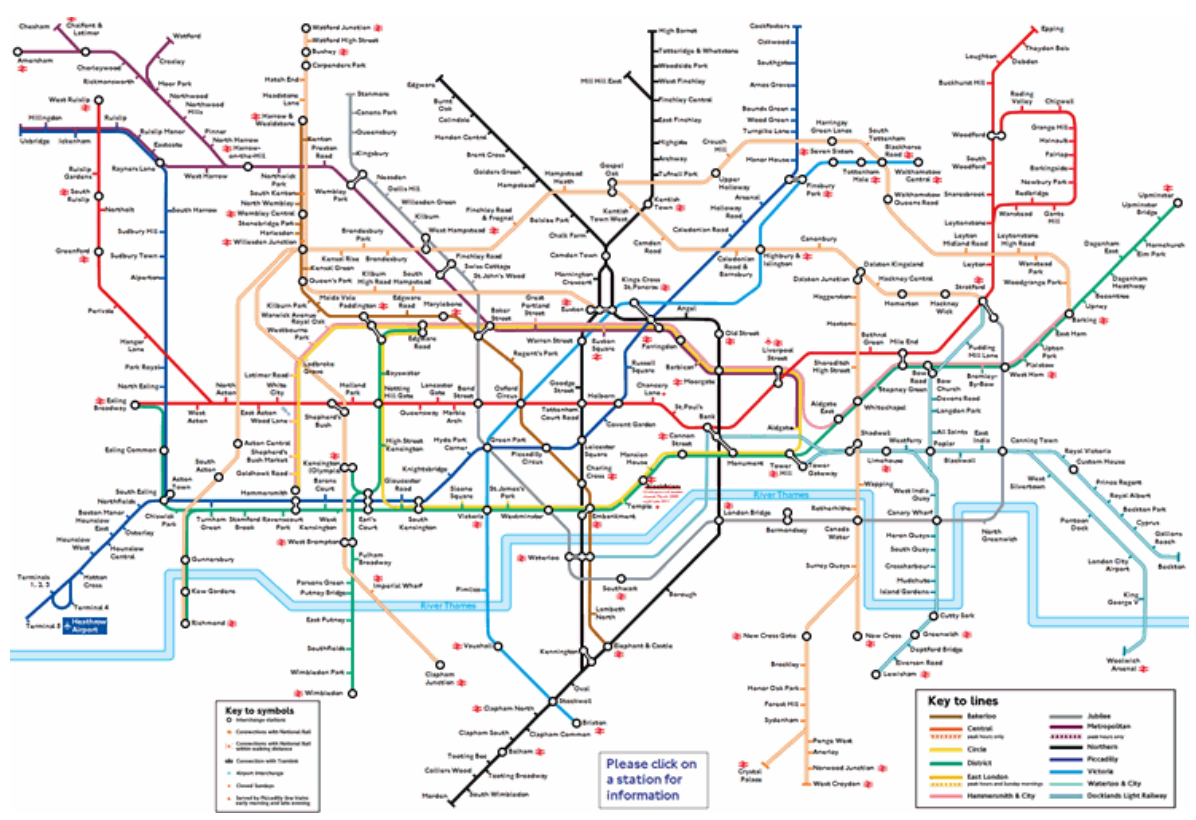

Figura 4. Mappa del sistema di trasporto metropolitano di Londra.

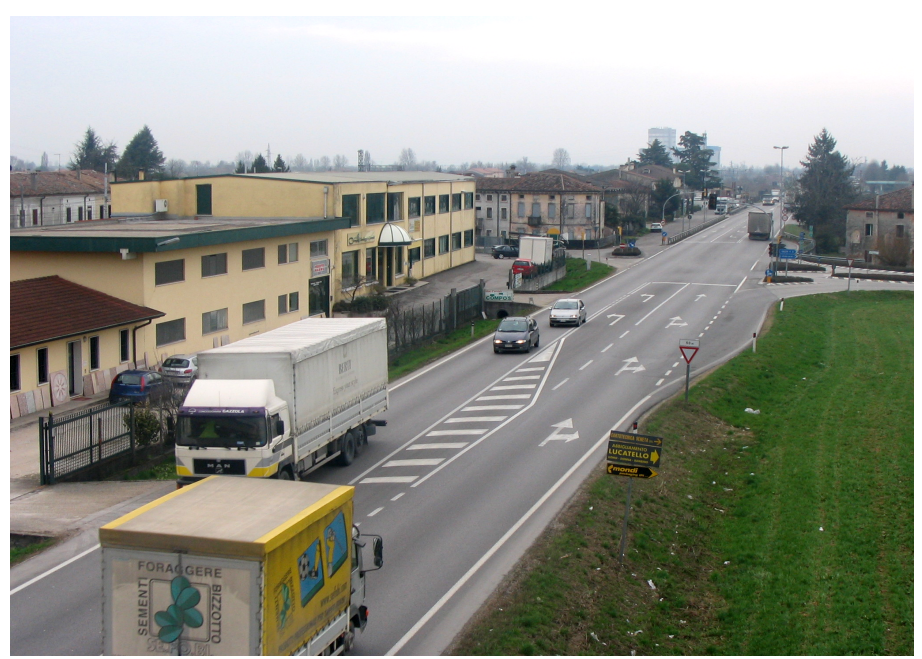

Figura 5. Veduta sulla Strada SS53, che collega le città di Vicenza e Treviso. Una strada-mercato, tipico asse della città diffusa del Veneto centrale. 


\section{Ocula ${ }^{14}$}

Le città visibili. Per Giovanna Franci | Elena Lorenzetto, Città o evento?

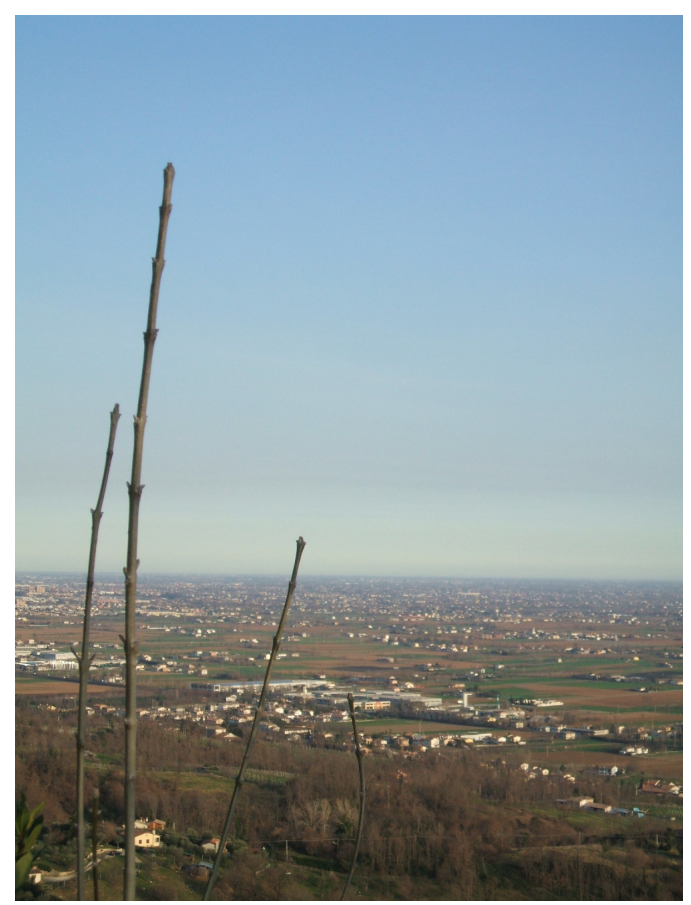

Figura 6. Veduta sull'area della Provincia di Treviso, dalla quale si può cogliere la dispersione urbana del territorio.

Si può considerare il Festival stesso come un discorso cartografico che riscrive il ritmo spaziale, le salienze e i punti di riferimento (cfr. Barthes 1985). Alle unità amministrative identificate come Comuni si tendono a sostituire luoghi della città diffusa resi pertinenti in quanto simbolo del Nord Est. Viene ridisegnato un paesaggio nuovo, giocando sulla neutralizzazione di tratti prima salienti come la campagna e gli elementi rurali, e sull'esaltazione di elementi a lungo rimossi nelle rappresentazioni tradizionali. Fabbriche, aree industriali dismesse, architetture contemporanee a uso aziendale sono rifunzionalizzate dal festival come luoghi culturali a fianco di palazzi storici e piazze.

Come ricorda Ugo Volli (2005), la città con la propria forma e struttura dirige lo sguardo dell'utente e in un certo senso lo genera. E una nuova città comporta anche la definizione di nuove categorie di percezione e lettura di essa. È quanto possiamo osservare nel caso del festival, il quale attraverso il suo kit di mappe, immagini e programmi costituisce una strategia di manipolazione dello sguardo.

\section{Il Festival come strategia territoriale}

L'evento culturale così inteso si può quindi considerare come un nuovo strumento non solo di marketing territoriale ma anche di pianificazione urbana, che mette in atto una strutturazione strategica dello spazio. Nel caso della città diffusa veneta questa operazione è ancora più forte e sentita. Parliamo infatti di un'area che negli ultimi trent'anni si è costituita per singole 


\section{Ocula $a^{14}$}

Le città visibili. Per Giovanna Franci | Elena Lorenzetto, Città o evento?

aggiunte e tattiche individuali, secondo una dinamica socio-economica dal basso, che Aldo Bonomi (1997) ha chiamato "capitalismo molecolare". Si potrebbe a questo proposito parlare di un vero e proprio evento modellizzante, che territorializza lo spazio, come abbiamo visto, in diversi modi: attraverso l'iscrizione di un insieme di funzioni prescritte, rappresentate dal palinsesto di eventi; attraverso una centralizzata e integrata strategia di enunciazione, come è evidente nell'analisi delle mappe del festival e della costruzione dell'identità "Nordest"; attraverso la riscrittura del territorio secondo un nuovo ritmo di elementi marcati e non marcati; e infine attraverso la costruzione di uno sguardo secondo certe categorie di lettura e percezione.

Possiamo cogliere l'efficacia del Festival, come strategia territoriale, nel fatto che in esso convivono sistema e uso. In altre parole, può essere visto come una sorta di fermo immagine sul processo dinamico di prassi enunciativa, descritto da Hjelmslev (1968), ossia il processo con cui si interdefiniscono, costituendosi reciprocamente, i diversi momenti del linguaggio: schema, norma, uso, parole. Infatti, sulla base di quanto illustrato, possiamo considerare il sistema del Festival come uno schema formale di città, basato su certi tratti formali e su una specifica strutturazione. Schema che è reso uso collettivo con lo svolgersi del grande evento: il pubblico "mette in pratica" questa forma, ossia la realizza localmente e individualmente attraverso la propria fruizione del festival. Viceversa il "fare la città" del festival, attraverso una pratica collettiva e un discorso messo in scena, può codificarsi in un sistema territoriale, dunque in un modello formale.

\section{Il caso di "Comodamente. Città e Parole in Festival"}

L'evento culturale mostra tutta la sua efficacia in questo senso, nel caso, come quello presentato, di città diffuse che mancano di un sistema. Tuttavia, si ritrovano tendenze analoghe anche in altri festival di taglia diversa e con strategie enunciative differenti. Per esempio, un caso altrettanto emblematico è Comodamente, festival annuale che si svolge dal 2007 a Vittorio Veneto e che propone tre giorni di dibattiti e spettacoli. Vittorio Veneto è un centro urbano di piccole dimensioni, in provincia di Treviso, con un centro storico concentrato, quindi presenta caratteri opposti a quelli delle "città impresa". La kermesse è stato ideata dal Centro Studi Usine, lo studio di progettazione urbana che è stato impegnato in una attività di riqualificazione urbanistica del centro storico, rispetto al quale il festival ha la funzione di "innovatore urbano", come dichiara il materiale informativo. Attraverso il proprio programma di eventi, l'intento è quello di vitalizzare i luoghi appena riqualificati e restaurati, inducendo la comunità a nuovi usi, percorsi e percezioni della città. I luoghi del festival sono allestiti con divani, tappeti, teli colorati e con una particolare cura nell'illuminazione (v. fig. 7), riconfigurando così il centro storico in un "salotto urbano", come enfatizza il materiale promozionale, in cui la comunità si senta comoda come a casa. Oltre agli spazi storici, quali piazze e palazzi, sono scelti come location anche spazi industriali dismessi ed edifici produttivi. L'evento diventa così motivo per rendere agibili luoghi dismessi, conferendovi nuovo o altro senso e valore. Agendo così sia sulla mor- 


\section{Ocula ${ }^{14}$}

Le città visibili. Per Giovanna Franci | Elena Lorenzetto, Città o evento?

fologia che sullimmaginario, il programma del festival diventa una nuova mappa della città.

Anche in questo caso si può osservare come l'evento costituisca una strategia di manipolazione volta a tradurre uno schema formale di città in uso collettivo e in prassi individuale e quotidiana. Per di più, questo caso esplicita come per ri-creare la città nelle sue molteplici dimensioni gli strumenti tradizionali dell'urbanistica necessitino di essere integrati ad altri supporti.

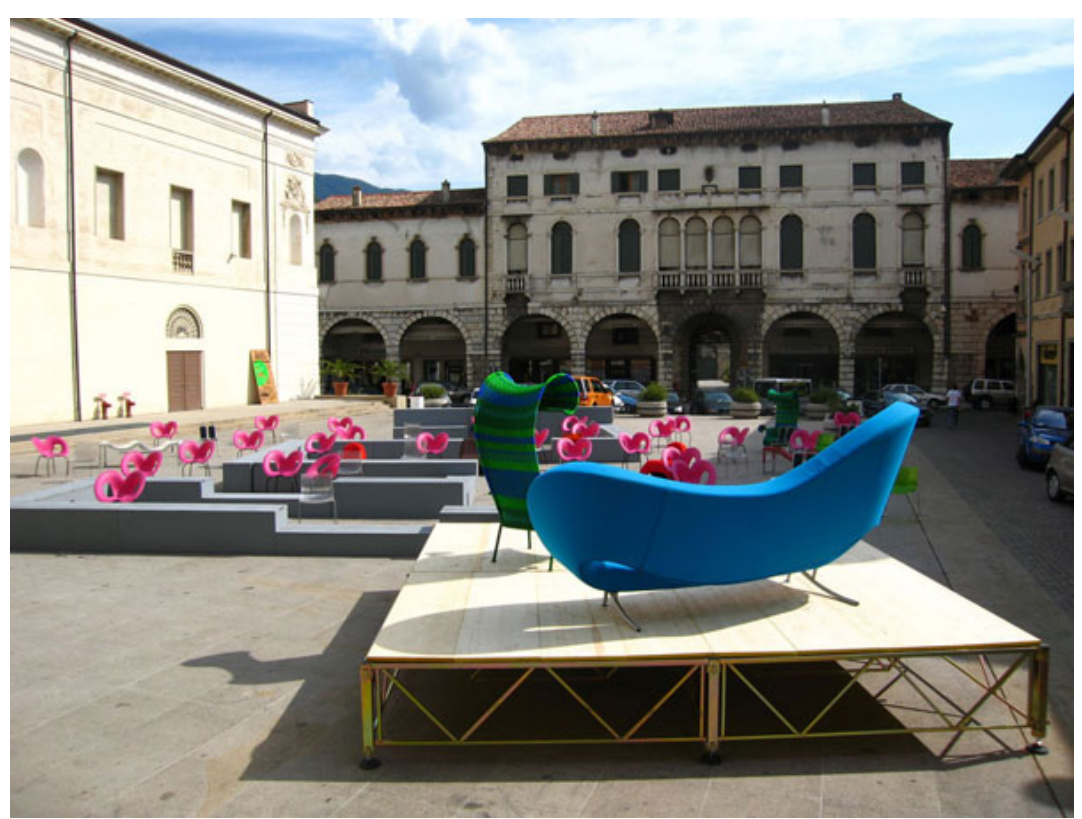

Figura 7. L'allestimento del festival Comoda_mente a Piazza Minuccio Minucci a Vittorio Veneto.

\section{Conclusioni}

Abbiamo quindi tutte le condizioni per attribuire al festival la funzione che Franco Farinelli (2003) riconosce al supporto cartografico: quella di dispositivo ontologico. Il festival crea la città che rappresenta. Se da una parte questo evento è fortemente subordinato ai luoghi, dall'altra il festival stesso è una nuova forma di territorio, che condensa in sé morfologia e discorso, sistema e processo.

Di conseguenza, questo dispositivo propone anche un nuovo concetto di territorio, di natura processuale, che si struttura nel suo farsi, in cui il fare città corrisponde alla messa in scena di un discorso, a partire da certe categorie di lettura, escludendo certi tratti e includendone altri, rendendo dominanti alcune isotopie.

Emerge però anche un aspetto critico dello strumento festival - sempre più evidente in questi ultimi anni in cui questa formula inizia a mostrare $\mathrm{i}$ propri limiti, anche economici. Lo si può riassumere nel fenomeno di festivalisation della cultura (cfr. Argano et al. 2005), secondo il quale i festival tendono a cannibalizzare la cultura. Cosicché un concerto di Mozart finisce per 


\section{Ocula ${ }^{14}$}

Le città visibili. Per Giovanna Franci | Elena Lorenzetto, Città o evento?

divenire un festival su Mozart, uno spettacolo su Shakespeare si trasforma in un festival su Shakespeare e così via. Anche spazi pubblici e urbani sono sempre più di frequente definiti sulla base di eventi di questo tipo.

Un'analoga festivalizzazione dei luoghi può portare a effetti differenti, che andranno a mio parere di volta in volta determinati e situati localmente, sulla base di variabili che distinguono un evento da un altro, quali le strategie enunciative $\mathrm{e}$ i valori in gioco. Si può però rilevare una tendenza comune, che consiste in una neutralizzazione dell'ordinario, dell'informale, dell'aspetto iterativo, a favore dello straordinario e dell'aspetto temporale singolativo. Il festival tende a istituire un nuovo criterio di visibilità e a costituire un nuovo punto di vista: il tempo festivaliero tende ad articolare il territorio e la città in luoghi visibili e invisibili, luoghi che parlano e luoghi muti, luoghi allestiti e luoghi vuoti, con il rischio di oscurare contenuti e forme dei luoghi esclusi. Inoltre, a identificare lo spazio della vita pubblica tendono a essere non tanto o non più i caratteri formali del luogo, bensì la pratica che vi si svolge e la temporalità del grande evento. Quindi, questo fenomeno emergente mette in discussione temi rilevanti, quali il ruolo della cittadinanza trasformata in pubblico, il valore del tempo del lavoro, le nuove forme di socialità. Questioni cruciali per chi si occupa di città e questioni in cui il margine tra città e rappresentazione mostra tutta la sua problematicità. 


\section{Ocula ${ }^{14}$}

Le città visibili. Per Giovanna Franci | Elena Lorenzetto, Città o evento?

\section{Bibliografia}

Argano, L. et al.

2005 Gli eventi culturali. Ideazione, progettazione, marketing, comunicazione, Milano, Franco Angeli editore.

Agusto, G.

2008 "Se il festival è di impatto. Gli effetti economici degli eventi festivalieri", Ticonzero, 88, 2008, www.ticonzero.info

Barthes, R.

1967 Sémiologie et urbanisme, in L'aventure sémiologique, Paris, Seuil, 1985 (tr. it. Semiologia e urbanistica, in L'avventura semiologica, Torino, Einaudi, 1991).

Bonomi, A.

1997 Il capitalismo molecolare. La società al lavoro nel nord Italia, Torino, Einaudi.

Farinelli, F.

2003 Geografia. Un’introduzione ai modelli del mondo, Torino, Einaudi.

Greimas, A. J.

1976 Por une sémiotique topologique, in Sémiotique et sciences sociales, Paris, Seuil (tr. it. Per una semiotica topologica, in Semiotica e Scienze sociali, Torino, Centro Scientifico Editore, 1991).

Guerzoni, G.

2008 Effettofestival. L'impatto economico dei festival di approfondimento culturale, La Spezia, Fondazione Carispe.

Hjelmslev, L.

1968 I fondamenti della teoria del linguaggio, Torino, Einaudi.

Indovina F. et al.

1990 La città diffusa, Venezia, DAEST.

Langen, F.; Garcia, B.

2009 "Measuring Impacts of Cultural Events Impacts", Impacts, 08, May 2009, www.impactso8.net.

Lorenzetto, E.

2012 "Manifesto per la metropoli del Nordest. Discorsi, immagini, eventi che fanno metropoli”, in Antonelli, R. e Macioti, I. M. (a cura di), Metamorfosi. La cultura della metropoli, Roma, Viella editrice.

Volli, U.

2005 Per una semiotica della città, in Laboratorio di semiotica, Bari-Roma, Laterza.

\section{Siti web}

www.festivaloffestivals.org

www.fanaticaboutfestivals.it

www.festivaldellecittàimpresa.it

www.comodamente.it 